\title{
Rôle accordé aux interactions entre pairs dans l'enseignement des mathématiques
}

Une illustration en classe d'accueil

Role given to peer interactions in mathematics education

An illustration of a reception class

\section{El rol acordado a las interacciones entre pares en la enseñanza de las matemáticas} Un ejemplo en clase de acogida

Louise Poirier

Volume 25, numéro 1, printemps 1997

L'apprentissage et l'enseignement des sciences et des mathématiques dans une perspective constructiviste

URI : https://id.erudit.org/iderudit/1080650ar

DOI : https://doi.org/10.7202/1080650ar

Aller au sommaire du numéro

Éditeur(s)

Association canadienne d'éducation de langue française

ISSN

0849-1089 (imprimé)

1916-8659 (numérique)

Découvrir la revue

Citer cet article

Poirier, L. (1997). Rôle accordé aux interactions entre pairs dans

l'enseignement des mathématiques : une illustration en classe d'accueil.

Éducation et francophonie, 25(1), 70-84. https://doi.org/10.7202/1080650ar
Résumé de l'article

Cet article a pour objectif d'illustrer un aspect du constructivisme, l'apport des interactions entre pairs dans l'enseignement des mathématiques. L'expérimentation s'est tenue dans une classe d'accueil du deuxième cycle du primaire. Après avoir présenté la problématique de la classe d'accueil et la théorie sous-jacente à l'approche, nous verrons comment, lors d'une séquence d'enseignement sur les fractions, s'est installée une culture de classe basée sur la discussion, l'argumentation, la négociation entre les élèves permettant à ces derniers de développer le sens de la fraction tout en approfondissant leur connaissance du français. Des extraits d'échanges entre élèves viendront illustrer nos propos.
Tous droits réservés (c) Association canadienne d'éducation de langue française, 1997 services d’Érudit (y compris la reproduction) est assujettie à sa politique d'utilisation que vous pouvez consulter en ligne. 


\section{Rôle accordé aux interactions entre pairs dans l'enseignement des mathématiques Une illustration en classe d'accueil}

\section{Louise POIRIER}

Université de Montréal, Québec, Canada

\section{RÉSUMÉ}

Cet article a pour objectif d'illustrer un aspect du constructivisme, l'apport des interactions entre pairs dans l'enseignement des mathématiques. L'expérimentation s'est tenue dans une classe d'accueil du deuxième cycle du primaire. Après avoir présenté la problématique de la classe d'accueil et la théorie sous-jacente à l'approche, nous verrons comment, lors d'une séquence d'enseignement sur les fractions, s'est installée une culture de classe basée sur la discussion, l'argumentation, la négociation entre les élèves permettant à ces derniers de développer le sens de la fraction tout en approfondissant leur connaissance du français. Des extraits d'échanges entre élèves viendront illustrer nos propos. 


\section{ABSTRACT}

\section{Role given to peer interactions in mathematics education - an illustration of a reception class}

Louise POIRIER

University of Montréal, Québec, Canada

This article is intended to illustrate one aspect of constructivism: the contribution of interactions between peers in the teaching of mathematics. The experiment was carried out in an integration class in the second cycle at the elementary level. After introducing the problem at the integration class and the theory underlying the approach, we shall see how, during the teaching of fractions, a class culture was developed, based on discussion, arguments and negociations among the students. This allowed them to comprehend the meaning of fractions while increasing their knowledge of French. Extracts from inter-student exchanges will serve to illustrate our text.

\section{RESUMEN}

\section{El rol acordado a las interacciones entre pares en la enseñanza de las matemáticas -un ejemplo en clase de acogida}

Louise POIRIER

Universidad de Montreal, Quebec, Canadá

Este artículo tiene como objetivo ilustrar un aspecto del constructivismo: la aportación de las interacciones entre pares en la enseñanza de las matemáticas. La experimentación se realizó en una clase de integración de alumnos inmigrantes del segundo ciclo de primaria. Debutaremos presentando la problemática específica de las clases de integración y de la teoría subyacente para después ver cómo, durante un periodo de enseñanza de las fracciones, se instaló un cultura de clase basada en la discusión, la argumentación y la negociación entre los alumnos, lo que les permitió desarrollar el significado de la fracción al mismo tiempo que mejoraban su conocimiento del francés. Algunos extractos de diálogo entre los alumnos ilustrarán nuestro tema. 


\section{Problématique}

La classe d'accueil vise l'intégration de l'élève immigrant allophone au milieu francophone et à la classe ordinaire. Cette intégration se fera principalement par l'apprentissage du français; d'autant plus que l'apprentissage des autres matières à l'étude devrait se faire dans cette nouvelle langue. Comment favoriser l'apprentissage des mathématiques dans un tel contexte? Deux types de caractéristiques liées à l'élève et à sa motivation, de même qu'à son milieu d'apprentissage, influencent l'apprentissage mathématique de l'élève qui arrive en classe d'accueil. Parmi les caractéristiques associées à l'élève, mentionnons d'abord sa maîtrise des mathématiques. Ainsi, un élève qui a eu certains contacts avec les mathématiques dans son pays d'origine a pu assimiler les concepts qui fondent cette matière et le transfert dans sa nouvelle langue se fera plus facilement. Une maîtrise minimale de la langue française peut aussi faciliter son apprentissage des mathématiques. Ces deux caractéristiques, on s'en doute, sont hors du contrôle de l'enseignante ou de l'enseignant, qui devra composer avec des élèves aux profils mathématiques et langagiers très diversifiés. Finalement, la motivation de l'élève a un impact sur son apprentissage. Notons que cette caractéristique n'est pas propre aux élèves de classes d'accueil. On parle ici plus spécifiquement de l'attitude de l'élève à l'égard des mathématiques, attitude qui peut être influencée par celle de ses parents et par sa culture face aux mathématiques ainsi que par l'attitude de l'enseignant et le type d'enseignement proposé.

Peuvent aussi favoriser l'apprentissage des mathématiques, des caractéristiques liées au milieu d'apprentissage, c'est-à-dire le développement d'une démarche propre à l'apprentissage des mathématiques en langue seconde qui met l'accent autant sur le vocabulaire approprié et la communication que sur la construction des concepts mathématiques par l'élève. Notre action se situe ici dans la mise en place de situations d'enseignement signifiantes pour le contexte de la classe d'accueil.

Avant d'entreprendre une telle démarche, il nous a semblé important, dans un premier temps, d'observer le fonctionnement de classes d'accueil afin de mieux en saisir le contexte et de mieux adapter nos interventions. Pour ce faire, nous avons assisté pendant un an (1993-1994) à plusieurs leçons de mathématiques dans diverses classes d'accueil. Nous avons alors été en mesure de dégager deux grands types d'enseignement des mathématiques alors en vigueur en classe d'accueil.

Une première pratique veut que l'enseignant familiarise d'abord les élèves avec le vocabulaire mathématique, souvent à l'aide d'illustrations représentant les nombres ou les figures géométriques, par exemple. Les élèves doivent alors apprendre le vocabulaire et associer le mot à l'objet mathématique. C'est comme s'il fallait connaître les mots et les termes appropriés avant de faire des mathématiques. Nous remettons en question ce type d'enseignement qui met l'accent sur les termes mathématiques avant de travailler les concepts. Nous soutenons l'idée inverse: nous pouvons mettre l'élève en situation de telle sorte qu'il puisse développer les concepts mathématiques sur lesquels nous viendrons greffer les termes appropriés.

Une deuxième pratique, liée à la diversité des groupes, caractérise la réalité de la classe. En effet, nous retrouvons dans la classe d'accueil des élèves d'âges variés 
(de 9 à 12 ans, par exemple). Non seulement ces élèves sont-ils d'âges différents, mais leur expérience mathématique antérieure l'est tout autant, certains d'entre eux ayant peu ou pas d'expérience scolaire (ce sont les élèves sous-scolarisés). La classe d'accueil est donc composée d'élèves d'âges variés, de cultures et de langues diverses, venant de systèmes scolaires tout aussi variés. Devant cette disparité des acquis mathématiques des élèves de la classe d'accueil, et soucieux du contenu mathématique à travailler avec ses élèves et de l'arrimage de ce contenu avec le programme du régulier, l'enseignant subdivise sa classe en autant de sous-groupes qu'il y a de niveaux scolaires présents. Chaque élève d'un sous-groupe aura son manuel scolaire, approprié à son niveau, et l'enseignant suivra ces différents manuels, donnant à chaque sous-groupe une série d'exercices (souvent du type exercice de calcul, application d'algorithme) à faire, puis il ira de sous-groupe en sous-groupe afin de donner des explications supplémentaires. Une telle pratique donne rapidement à l'enseignant une impression d'essoufflement; il est débordé et n'a pas de vue d'ensemble de sa classe. Les élèves, pour leur part, n'ont plus le sentiment de faire partie d'une classe, mais plutôt d'un sous-groupe basé sur leur compétence (ainsi un élève sousscolarisé de 12 ans peut se retrouver avec les plus jeunes de la classe, qui sont des enfants de deuxième année). Il en résulte moins d'occasions d'interaction avec le groupe-classe, moins de communication. Or, l'apprentissage ne se fait pas de façon isolée, mais par le biais de situations sociales où le langage est utilisé dans un processus de dialogue (Vygotsky, 1978). Sans totalement rejeter ce type d'exercice individuel, les élèves en ayant besoin afin de consolider leurs apprentissages, nous ne pouvons bâtir notre enseignement autour de ces seules activités.

La démarche que nous proposons mise sur les interactions entre pairs, sur la communication pour, d'une part, développer les concepts mathématiques et, d'autre part, apprendre à communiquer en français. Cette approche puise ses fondements théoriques dans la perspective socioconstructiviste. Dans le présent article, nous mettrons l'accent sur un aspect du socioconstructivisme, le rôle des interactions sociales, et nous nous centrerons sur la construction de concepts mathématiques par les élèves. Sans vouloir minimiser les acquisitions langagières des élèves, nous en parlerons à l'occasion, le but premier de notre intervention étant de mettre en place des activités signifiantes pour le développement mathématique.

\section{Contexte théorique sous-jacent}

La dimension sociale du développement mathématique a pris une importance croissante dans les réflexions sur l'enseignement des mathématiques (Ernest, 1991; Bauersfeld, 1988). Lakatos (1976) dit des mathématiques qu'elles sont un dialogue entre des individus aux prises avec des problèmes mathématiques à résoudre. Cette dimension sert de cadre de référence à des activités menées en classe avec les élèves (Poirier et Bacon, 1996; Cobb, Yackel et Wood, 1992; Bednarz, 1991) où les processus de dialogue et de critique qui sont à la base du développement mathématique lui-même 
jouent un rôle de première importance dans la construction de la connaissance mathématique.

\section{Les balises du socioconstructivisme}

Dans son livre The Philosophy of Mathematics Education paru en 1991, Ernest discute des balises du socioconstructivisme. S'inspirant des travaux de Wittgenstein et de Lakatos, il nous dit que pour le socioconstructivisme la connaissance mathématique est une construction sociale, puisque la base de la connaissance mathématique, la connaissance linguistique avec ses conventions et ses règles, est une construction sociale et que les processus sociaux interpersonnels de dialogue et de critique sont nécessaires pour changer la connaissance mathématique subjective d'un individu en une connaissance objective socialement acceptée.

Le point central du socioconstructivisme est la genèse de la connaissance, mathématique dans notre cas. Ainsi, une nouvelle connaissance mathématique peut être soit subjective (propre à un individu) ou objective (commune à un groupe). Ernest, dans son modèle, considère ces deux formes de connaissance et les relie dans un cycle où chacune contribue au renouveau de l'autre. Ce cycle va d'une connaissance subjective (création personnelle d'un individu) au moyen d'une publication à une connaissance objective, acceptée socialement. Cette connaissance objective est par la suite intériorisée, reconstruite par des individus durant leur apprentissage des mathématiques pour ainsi devenir une connaissance subjective. En utilisant cette connaissance, les individus créent et publient de nouvelles connaissances mathématiques, complétant ainsi le cycle. La figure qui suit, tirée du livre de Ernest, illustre cette relation entre connaissance objective et connaissance subjective.

Figure 1. Relation entre la connaissance objective et la connaissance subjective

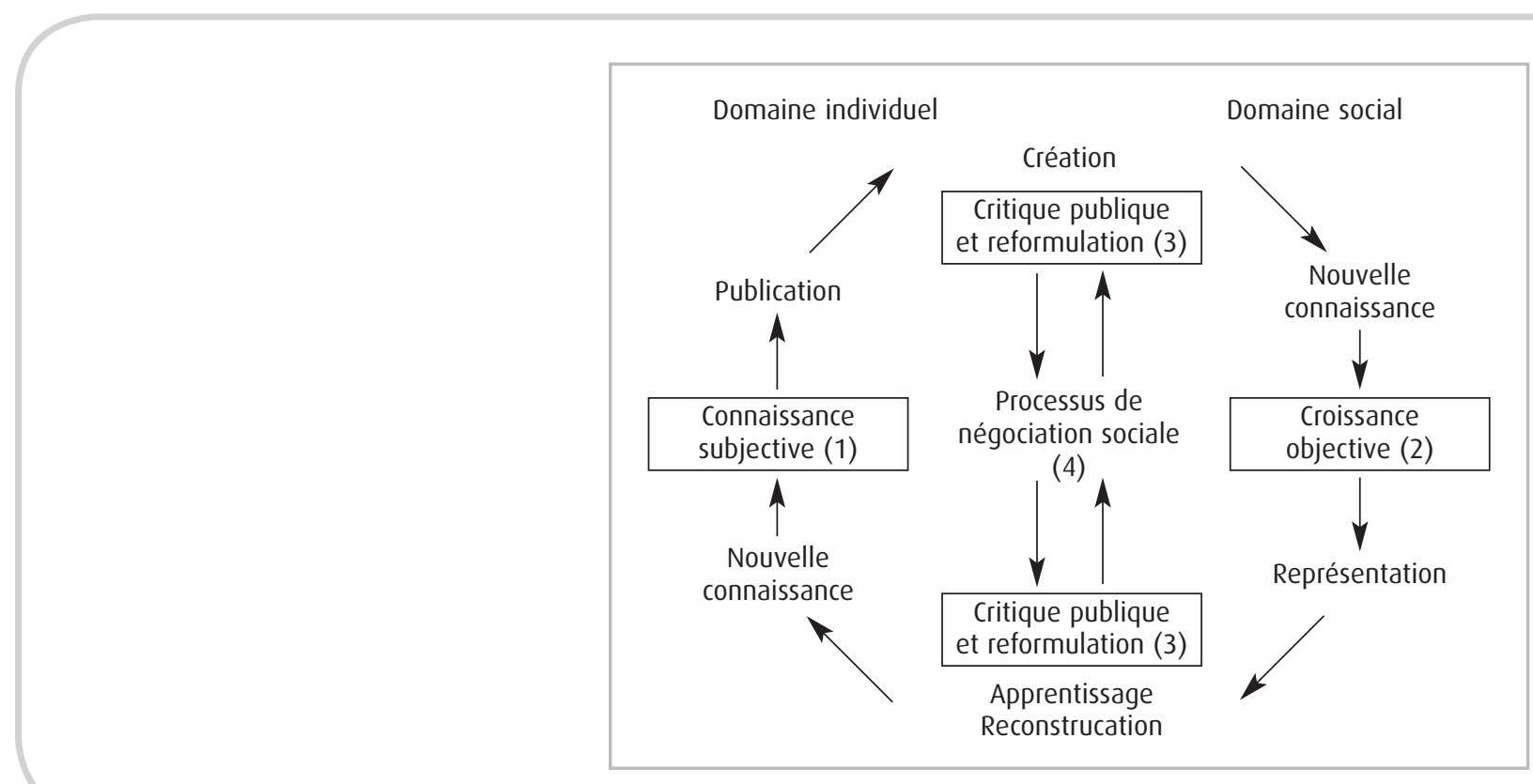


En reprenant la figure, nous voyons que tout individu possède des connaissances subjectives des mathématiques. La pensée fondamentale d'un individu est une pensée subjective (1). La connaissance mathématique est pour la plus grande part une connaissance objective apprise par un processus de re-création qui résulte en représentations uniques, subjectives de la connaissance mathématique. Pour qu'une telle connaissance subjective d'un individu devienne une connaissance objective (2), il faut d'abord la rendre publique (3), mais cela ne suffit pas. Il faut qu'elle soit socialement acceptée. Une connaissance rendue publique devient une connaissance objective par un examen critique des autres en suivant le processus heuristique proposé par Lakatos: hypothèse - preuve - émergence de contre-exemples - réexamen de la preuve - reformulation du théorème - ouverture à d'autres domaines de recherche... (4). Il peut donc résulter de cette nouvelle connaissance une acceptation, qui demeurera toutefois ouverte à la critique et à l'étude. Cette connaissance objective devient une connaissance subjective pour un autre individu (1). Cette nouvelle connaissance subjective est en grande part une connaissance objective re-construite et intériorisée. Cette intériorisation, ou représentation interne et subjective, est une étape cruciale du cycle de la création mathématique et l'enseignement doit lui porter un intérêt tout particulier.

\section{Comment un individu peut-il acquérir une connaissance du monde externe?}

Pour le socioconstructivisme, l'esprit humain est actif, il fait des prédictions, des hypothèses à partir de ses expériences du monde physique et social, construisant ainsi des théories, des modèles. Ces modèles lui servent de guide pour agir; lorsqu'ils s'avèrent inadéquats, ils sont reformulés et modifiés ou rejetés et remplacés. Deux principes fondamentaux émergent:

a. La connaissance n'est pas reçue passivement, mais est construite activement par le sujet.

b. La fonction de la cognition est adaptative et sert à organiser le monde.

Ernest rejoint en cela les propos de von Glasersfeld (1989) pour qui toute connaissance n'est pas reçue passivement, mais est une construction individuelle qui s'adapte à des contraintes. La connaissance prend ici son origine dans l'action du sujet qui apprend. L'action sur le monde physique et social devient essentielle à l'élève pour lui permettre d'accommoder ses schèmes et pour assimiler de nouvelles situations.

\section{Impact sur l'enseignement}

L'élève devra donc se trouver en situation stimulant ses propres activités, l'amenant à présenter et discuter ses idées, ses procédures, à argumenter, à négocier avec les autres élèves. Qu'entend-on par "situation»? Comme nous l'avons vu précédemment, pour Lakatos les mathématiques sont un dialogue entre individus aux prises avec des problèmes mathématiques. S'il n'y a pas de problèmes à résoudre, de défis à relever, il n'y aura aucune motivation à développer de nouveaux concepts. Le socioconstructivisme identifie les mathématiques en tant qu'institution sociale, résultant de 
l'émergence et de la résolution de problèmes. On attribue ici un rôle central aux problèmes. L'enseignement devra fournir aux élèves des situations problèmes riches et signifiantes permettant la discussion, l'argumentation, la négociation entre eux. L'expérience sociale est donc appelée à jouer un rôle fondamental, participant au développement cognitif de l'élève et à sa construction de connaissances mathématiques. Dans cette perspective, «les dynamiques individuelles sont conçues comme se fondant sur des expériences sociales qu'elles sont amenées à structurer» (Mugny, 1985, p. 18). Cette dynamique va être envisagée dans les recherches qui s'inscrivent dans cette perspective socioconstructiviste, sous différentes formes. À l'origine, elle renvoie aux interactions entre enfants, mettant en place un conflit sociocognitif, c'est-à-dire:

un conflit structurant, source de changement chez l'individu. Ce conflit ne sera possible que lors d'interactions où s'expriment simultanément plusieurs actions, solutions, discours... dans lesquels se manifeste un système de centrations cognitives opposées, posant le problème de leur coordination en de nouveaux systèmes d'ensemble qui permettent d'en rendre compte (Garnier, Bednarz et Ulanovskaya, 1991, p. 23).

Ayant comme point de départ le conflit sociocognitif, les recherches vont peu à peu s'en éloigner pour englober graduellement d'autres dimensions du social. C'est ainsi que des études s'intéressant au rôle des variables sociales dans le développement cognitif vont graduellement prendre place:

Dans ces recherches, l'expérience sociale est ainsi envisagée au-delà du conflit, sous les aspects signifiants attachés aux contenus des tâches et situations. Cette nature sociale de la situation présentée aux sujets rejoint les préoccupations de certains didacticiens sur le contrat didactique qui lie l'intervenant, les élèves et le savoir au sein d'une situation pédagogique (Brousseau, 1986; Schubauer-Leoni, 1986) (Garnier, Bednarz et Ulanovskaya, 1991, p. 23).

D'autres études vont s'interroger davantage sur le rôle des particularités culturelles et sociales dans la construction des connaissances mises en place (Nunès, 1991; Janvier, 1991; D’Ambrosio, 1989). Un dernier type de recherche va porter sur la dynamique des interactions sociales entre élèves qui effectuent ensemble une même tâche. C'est la perspective que nous reprenons ici.

Dans de telles études, les confrontations entre élèves, mises en place dans la classe et contribuant à l'évolution des connaissances mathématiques, peuvent prendre diverses formes: situations d'action entre les sujets dans lesquelles les élèves se partagent une même situation problème, des situations de communication entre deux sujets ou deux groupes qui ont des rôles sociaux différents (Laborde, 1991; Brousseau, 1986) et des situations de validation où différentes solutions ou procédures seront exprimées et discutées. Ces diverses confrontations entre les élèves jouent un rôle important dans la théorie des situations de Brousseau $(1972,1986)$ où ce dernier développe le processus de mathématisation. 
Durant la première phase du processus de mathématisation, nommée «dialectique de l'action", l'enfant est amené à élaborer certains modèles mentaux des relations entre les données de la situation, modèles qui guideront son action. C'est à partir de ces modèles que le processus d'apprentissage s'articule. Pour qu'il y ait mathématisation, il faut que l'élève soit en mesure d'expliciter son modèle par le biais d'un langage approprié. C'est la phase fondamentale chez Brousseau de la «dialectique de la formulation» «... il est clair qu'il n'y a pas vraiment apprentissage des mathématiques sans l'emploi par l'élève de modèles explicites, du langage et de l'écriture mathématique" (Brousseau, 1972, p. 64). Cette dialectique de la formulation met au premier plan les interactions entre les élèves. Durant ces interactions, les élèves doivent expliciter les termes utilisés, leurs choix; ceci entraîne des débats entre les élèves. Ces situations de débats, ou «dialectique de la validation", constituent l'occasion de discussions entre élèves comme moyen d'établir des preuves, d'en rejeter. «Faire des mathématiques ne consiste pas seulement à émettre ou à recevoir des informations en langage mathématique, même en les comprenant. L'enfant mathématicien doit prendre maintenant vis-à-vis des modèles qu'il a construits une attitude critique» (Brousseau, 1972, p. 64). Nous voyons dans ces différentes phases du processus de mathématisation l'importance accordée aux interactions entre élèves, au même titre que le processus de négociation sociale décrit par Ernest.

\section{L'approche mise en place}

L'intervention, mise en place à l'automne $1995^{1}$, s'est déroulée durant toute l'année scolaire. La classe comptait 16 élèves âgés de 9 à 12 ans, d'origines diverses (Roumanie, Pakistan, Égypte, Russie, Haïti, Liban, Sri Lanka, Guatemala, Cambodge). Quant à leurs niveaux scolaires, ils variaient de la deuxième à la sixième année du primaire. La plupart des élèves ont été scolarisés dans leur pays d'origine, allant parfois aussi à l'école dans un pays de transition avant d'arriver au Québec. Durant l'année scolaire, nous avons travaillé les notions mathématiques à l'étude au programme du deuxième cycle primaire. Toutes les séances en classe ont été filmées sur bandes vidéoscopiques, puis retranscrites sous forme de protocole. De plus, les productions des élèves, sous forme écrite, ont été conservées. Dans ce qui suit, nous allons illustrer la dynamique de la classe à partir du protocole d'une leçon portant sur les fractions, leçon qui s'est tenue au mois de février, soit au début de l'enseignement des fractions.

\section{Description de la séquence d'activités}

C'est par le pliage de bandes de papier (Scott, 1981) que les élèves ont d'abord été amenés à construire, comparer, ordonner des nombres fractionnaires. Les élèves ont aussi estimé, trouvé les facteurs d'un nombre. Une mise en contexte des bandes

1. Intervention mise en place dans la classe de madame Madeleine Duranleau, enseignante en classe d'accueil à l'école François-de-Laval, CECM. 
de papier a permis, par la suite, la résolution de problèmes écrits. Nous avons travaillé ici un des sens de la fraction, soit la partie d'un tout; d'autres sens de la fraction ont été vus dans des leçons subséquentes.

Après avoir distribué des bandes de papier de même longueur $(21,5 \mathrm{~cm}$ sur $3 \mathrm{~cm}$ ) à chaque élève, nous avons demandé à chacun de prendre une bande de papier et de la plier en deux parts égales. Tous les élèves, peu importe leur âge ou leur scolarité antérieure, peuvent plier une bande de papier en deux parts égales; tous les élèves de la classe sont alors engagés dans la même activité.

\section{Enseignante}

On a plié la bande de papier en deux. (Elle la déplie.) Combien de parts, combien de sections avez-vous?

Les élèves (en chœur)

Deux!

\section{Enseignante}

(Elle dessine un rectangle au tableau, le divise en deux et colorie une part.) Quelle portion de la bande de papier j'ai coloriée?

\section{Élève 1}

(Il écrit avec son doigt dans les airs $1 / 2$.)

\section{Enseignante}

Peux-tu venir l'écrire au tableau?

Élève 1

(Il écrit $1 / 2$. .)

\section{Enseignante}

Comment on dit ça?

\section{Élèves}

(Silence)

\section{Enseignante}

Avez-vous déjà vu ça?

\section{Élèves}

Une dizaine d'élèves lèvent la main.

\section{Enseignante}

En français, on dit demi (elle écrit au tableau une demie). J'ai colorié une demie de la bande de papier. On peut dire aussi la moitié. (Elle écrit moitié au tableau.)

Le même scénario est repris avec une autre bande de papier, cette fois pliée en quatre. L'extrait que nous venons de présenter illustre comment les termes mathématiques, une demie, un quart viennent se greffer, après coup, à l'action. Nous n’avons pas présenté aux élèves des rectangles dessinés et divisés en deux ou en quatre en leur disant voici une demie, voici un quart. Le pliage des bandes de papier a amené les élèves à construire une demie et un quart, que nous avons par la suite nommés. L'enseignante demande ensuite aux élèves de plier une autre bande de papier afin d'obtenir huit parts. La longueur de la bande de papier, soit 21,5 cm, ne permet pas aux élèves d'avoir recours aisément au mesurage et à la division 
$(21,5 \mathrm{~cm} \div 8)$. Aucun élève n'a d'ailleurs ressenti le besoin d'utiliser sa règle. C'est donc à nouveau par le pliage que les élèves sont arrivés, non sans difficulté, à obtenir huit parts égales.

\section{Plusieurs élèves}

C'est facile!

Élève 2

(Après avoir plié sa bande de papier en deux et encore en deux, l'élève la déplie, compte les parts obtenues et la replie encore en deux. Il dénombre maintenant 8 parts.) J'ai fini, Madame!

\section{Enseignante}

Lorsque les élèves ont terminé leur pliage, l'enseignante leur demande de vérifier s'ils ont vraiment 8 parts.

Élève 3

(Elle en dénombre 16.)

Élève 4

J'en ai 8

\section{Enseignante}

Comment as-tu fait?

Élève 4

J'ai plié en deux, toujours en deux.

\section{Enseignante}

Peux-tu me dire exactement ce que tu as fait? Je vais l'écrire au tableau.

Élève 4

J'ai plié en deux, puis j'ai plié en deux.

\section{Enseignante}

(Tout en écrivant) Combien de parts avais-tu alors?

Élève 4

Quatre parts, puis j'ai plié encore en deux. C'est comme 2 fois 2 fois 2 , ça fait 8.

Enseignante

C'est beau! Est-ce qu'il y en a qui ont fait cela d'une autre façon?

Élève 5

Moi, j'ai pris la bande de quatre et j'ai plié en deux.

\section{Enseignante}

(Elle écrit au tableau: bande de 4 pliée en 2.)

Élève 4

C'est comme 4 fois 2.

\section{Enseignante}

(S'adressant à l'élève 3 qui avait obtenu 16 parts)

Comment as-tu fait pour avoir 16 ?

Élève 3

(Reprenant sa bande et la repliant) J'ai plié ma bande, oui, en deux, puis j'ai plié en deux, en deux et en deux. (Procédure additive erronée, car elle a fait $2+2+2+2)$ 


\section{Élève 4}

Tu en as fait trop!

\section{Enseignante}

Qu'est-ce que tu as obtenu?

\section{Élève 3}

16. C'est trop. (Reprenant à son compte l'explication de l'élève 4, qui le premier a fait ressortir la structure multiplicative de la situation) J'ai fait $8 \times 2$, 16.

\section{Enseignante}

(Elle dessine un rectangle, le divise en 8 parts, en colorie une.) Comment on pourrait écrire une part?

\section{Élève 6}

(Il va au tableau et écrit 1/8.)

\section{Enseignante}

Comment on va dire cela?

\section{Élève 2}

Un octave.

L'extrait que nous venons de voir illustre plusieurs aspects de l'approche. Un tel pliage de bandes de papier fait appel à une structure multiplicative, comme l'exprime l'élève 4 . Or, il n'est pas rare de trouver dans une telle situation des élèves qui auront recours à une procédure additive erronée (voir l'élève 3). En provoquant l'utilisation de cette procédure additive, nous forçons cette élève à réorganiser sa pensée; ce qu'elle fait en s'appuyant sur l'argumentation de l'élève 4 qui a auparavant fait ressortir la structure multiplicative. C'est d'ailleurs la même élève qui, quelques minutes plus tard, ira montrer aux autres comment plier une bande de papier en cinq parts égales. De plus, ce fut une belle occasion de revenir sur l'étymologie du mot «octave» grâce à l'intervention de l'élève 2 qui propose le terme «octave» pour désigner la fraction 1/8. Les élèves sont ensuite amenés à plier une bande de papier en trois parts égales. Ce pliage s'est avéré beaucoup plus complexe pour certains élèves, puisqu'on ne peut, ici, se baser sur un pliage en deux. C'est un élève de la classe qui a trouvé une technique astucieuse en "roulant» la bande de papier de manière à obtenir trois épaisseurs. Il est alors invité à expliquer et démontrer sa technique aux autres. Ce dernier pliage en trois parts égales amène l'élève 1 à émettre une hypothèse:

Élève 1

C'est facile! Les nombres pairs, c'est toujours plier en deux. (N'oublions pas que, jusqu'ici, nous avons fait 2, 4, 8 et 3.) Les nombres impairs, c'est plus difficile.

\section{Enseignante}

Je vous demande maintenant de prendre une autre bande de papier et de la plier pour avoir 6 parts.

Élève 2

(Reprenant sa procédure de dénombrement au fur et à mesure, il plie la 
bande en deux puis en deux, ce qui lui donne quatre parts; la replie en deux et dénombre huit parts.)

\section{Élèves}

(Plusieurs élèves, par pliage, arrivent à 8 parts. Quelques élèves procèdent par pliage "accordéon", c'est-à-dire qu'ils font une première pliure à partir d'une extrémité de la bande, puis replient de la même largeur jusqu'à ce qu'ils atteignent l'autre extrémité de la bande. La largeur de la première pliure est aléatoire; c'est ainsi qu'ils arrivent à 4,5 ou 6 parts et une autre plus petite ou plus grande. Les élèves recommencent à plusieurs reprises pour trouver 6 parts.)

\section{Élève 1}

6, c'est pair, mais on peut pas!

\section{Élève 7}

(Se lève de sa chaise pour nous montrer son pliage.) Moi, Madame! Moi, Madame! J'ai trouvé! (Notons qu'il s'agit d'un élève sous-scolarisé.)

\section{Enseignante}

Qu'as-tu fait?

\section{Élève 7}

J'ai fait comme ça (et il nous montre un pliage en trois, puis un pliage en deux). Élèves

Plusieurs élèves reprennent sa façon de procéder.

\section{Enseignante}

Est-ce qu'il y aurait eu une autre façon de procéder?

\section{Élève 8}

On peut plier en deux, puis en trois.

\section{Enseignante}

(S'adressant à l'élève 7) Est-ce différent de ta façon de faire?

\section{Élève 7}

J'ai fait en trois et en deux. (On en profite pour discuter de la commutativité en multiplication.)

Dans cet extrait, on voit bien la dynamique de la classe qui s'est installée. Les élèves, depuis le début de l'année scolaire, sont amenés à émettre des hypothèses, à argumenter, à vérifier. C'est ainsi que l'élève 1 nous dit que le pliage de nombres pairs est facile, puisque c'est toujours par deux. L'enseignante leur propose alors un nombre pair, 6, mais qui ne pourra se faire uniquement en pliant par deux. Il est intéressant de noter que le premier élève à trouver la procédure est un élève très peu scolarisé dans son pays d'origine. Nous revenons ici sur un des buts de l'activité, à savoir la participation de tous les élèves de la classe peu importe leur niveau scolaire. Cet épisode de l'activité a permis un premier travail sur les facteurs. L'enseignante en profitera ensuite pour demander aux élèves de trouver différentes façons d'obtenir 12 parts, sans faire le pliage. En d'autres termes, les élèves sont-ils capables, à ce point de l'activité, de se détacher du pliage et de dégager la structure multiplicative afin de trouver les facteurs de 12 ? 
L'activité se poursuit par des pliages en 9 et en 5 . Partant de leur pliage en 3, des élèves arrivent à la conclusion que pour trouver 9 parts il faut plier en 3 et replier de la même façon en 3 . Le pliage en 5 , nombre premier, s'avère plus difficile: il n'y a qu'une façon de plier en 5 , et cela demande une estimation à partir des bandes de 4 et de 6 déjà faites. Les élèves ont donc sur leur pupitre des bandes de papier pliées en $2,3,4,5,6,8$ et 9. L'enseignante leur demande de les placer en ordre croissant de la plus petite part à la plus grande part. Les élèves y parviennent, non sans difficultés; le nombre 2 est plus petit que 3 et, pourtant, une demie, c'est plus grand qu'un tiers... Une discussion s'engage alors entre les élèves.

Élève 2

J'ai compris qu'en 2, c'est plus grand qu'en 3, plus grand qu'en 4, toujours plus grand.

\section{Élève 9}

(Reprenant ce que l'élève 2 a dit) Oui, je comprends que plus le chiffre est petit plus la partie est grande.

Élève 2

C'est ça!

Élève 10

Plus le nombre de parties est petit, plus chaque partie est grande.

Cet extrait nous montre comment l'explication partielle du premier élève ( «en 2 , c'est plus grand qu'en $3 »)$, reprise et raffinée par deux autres élèves, devient un principe plus général: "plus le nombre de parties est petit, plus chaque partie est grande». L'enseignante en profite alors pour introduire l'idée du numérateur et du dénominateur ainsi que leur rôle respectif.

\section{Conclusion}

Les bénéfices que l'élève peut tirer des interactions sociales sont nombreux et se situent à divers moments du processus, reformulation par un élève de la consigne donnée par l'enseignante, explicitation de la démarche, d'une stratégie, d'une technique, débat autour d'une hypothèse, d'une solution... À chacun de ces moments, le fait de communiquer permet à l'élève d'affiner sa pensée; cela permet aussi aux autres élèves de s'approprier une démarche, une justification et, finalement, cela permet une coconstruction des concepts mathématiques.

En choisissant de travailler les fractions, nous avions un défi de taille à relever. En effet, nous voulions illustrer comment il est possible de travailler en classe d'accueil, avec des élèves d'âges et de niveaux scolaires différents, à une tâche commune. Bien que les élèves aient eu l'âge correspondant au deuxième cycle du primaire, cycle où sont introduites les fractions, certains d'entre eux avaient peu ou pas d'expérience scolaire antérieure (par le fait même peu ou pas d'expérience avec les fractions). C'était le cas, entre autres, des élèves 1 et 7; pourtant, ces deux élèves ont été des facteurs importants dans la dynamique de cette leçon et ont grandement aidé, par leurs 
interventions, la coconstruction d'un sens de la fraction. En misant sur l'importance des interactions sociales et en mettant les élèves en situation riche et signifiante, l'enseignante les a amenés à expliciter, à justifier leurs choix, à débattre des idées leur permettant ainsi de construire des concepts mathématiques. De plus, nous sommes dans une classe d'accueil, en février, et ces élèves ont au mieux six mois d'expérience en français; l'approche leur a aussi permis de s'exprimer et de débattre en français, d'apprendre, en contexte, de nouveaux termes. L'extrait présenté ici illustre aussi le rôle important de l'enseignant tant lors de la planification des activités, par les choix didactiques à poser (par exemple, la dimension des bandes de papier), que lors de la réalisation des activités en classe. Tout au long de la leçon, le questionnement de l'enseignant doit s'articuler sur la démarche des élèves afin de leur permettre d'affiner leur pensée et d'argumenter. Cette année, l'expérience continue avec la même enseignante, de façon systématique, afin de mieux suivre le développement mathématique et langagier de chaque élève. L'expérience poursuit deux buts: développer des activités et une approche d'enseignement adaptées à ces élèves et mieux comprendre les éléments en jeu dans un tel contexte.

\section{Références bibliographiques}

BAUERSFELD, H. (1988). Interaction, construction and knowledge. Alternative perspectives for mathematics education. Dans T. Cooney et D. Grouws (dir.), Effective Mathematics Teaching. Reston, VA: National Council of Teachers on Mathematics.

BEDNARZ, N. (1991). Interactions sociales et construction d'un système d'écriture des nombres en classe primaire. Dans C. Garnier, N. Bednarz et I. Ulanovskaya (dir.), Après Vygotski et Piaget. Perspectives sociale et constructiviste. Écoles russe et occidentale (p. 51-67). Bruxelles: De Boeck.

BROUSSEAU, G. (février 1972). Processus de mathématisation. Bulletin de l'Association des professeurs de mathématiques de l'enseignement public, $\mathrm{n}^{\mathrm{o}}$ 282, 57-84.

BROUSSEAU, G. (1986). Théorisation des phénomènes d'enseignement des mathématiques. Thèse de doctorat d'État. Université de Bordeaux.

COBB, P., YACKEL, E. et WOOD, T. (1992). A constructivist alternative to the representational view of mind in mathematics education, Journal for Research in Mathematics Education, 23(1), 2-33.

D'AMBROSIO, V. (1989). What can we expect from ethnomathematics? Dans C. Kertel (dir.), Science and Technology Education. Mathematics, Education and Society. Paris: UNESCO. 
ERNEST, P. (1991). The Philosophy of Mathematics Education. Hamshire: The Falmer Press.

GARNIER, C., BEDNARZ, N. et ULANOVSKAYA, I. (dir.) (1991). Après Vygotski et Piaget. Perspectives sociale et constructiviste. Écoles russe et occidentale. Bruxelles: De Boeck.

GLARSERSFELD, E. von (1989). Constructivism in education. Dans T. Husen et N. Postlethwaite (dir.), International Encyclopedia of Education (p. 162-163). Oxford: Pergamon.

JANVIER, C. (1991). Contextualisation et représentation dans l'utilisation des mathématiques. Dans C. Garnier, N. Bednarz et I. Ulanovskaya (dir.), Après Vygotski et Piaget. Perspectives sociale et constructiviste. Écoles russe et occidentale. Bruxelles: De Boeck.

LABORDE, C. (1991). Deux usages complémentaires de la dimension sociale dans les structures d'apprentissage en mathématiques. Dans C. Garnier, N. Bednarz et I. Ulanovskaya (dir.), Après Vygotski et Piaget. Perspectives sociale et constructiviste. Écoles russe et occidentale. Bruxelles: De Boeck.

LAKATOS, I. (1976). Proofs and Refutations. The Logic of Mathematical Discovery. Cambridge, MA: Harvard University Press.

MUGNY, G. (1985). Psychologie sociale du développement cognitif. Berne: Peter Lang.

NUNÈS, T. (1991). Systèmes alternatifs de connaissances selon différents environnements. Dans C. Garnier, N. Bednarz et I. Ulanovskaya (dir.), Après Vygotski et Piaget. Perspectives sociale et constructiviste. Écoles russe et occidentale. Bruxelles: De Boeck.

POIRIER, L. et BACON, L. (1996). Interactions between children in mathematics class. An example concerning the concept of number. Dans H. Mansfield, N. A. Pateman et N. Bednarz (dir.), Mathematics for Tomorrow's Young Children (p. 166-176). Dordrecht: Kluwer Academic Publishers.

SCHUBAUER-LEONI, M. L. (1986). Le contrat didactique: un cadre interprétatif pour comprendre les savoirs manifestés par les élèves en mathématique. European Journal of Psychology of Education 1(2), 139-153.

SCOTT, W. R. (janvier 1981). Fractions taught by folding paper strips. The Arithmetic Teacher, 28(5), 18-21.

VYGOTSKY, L. S. (1978). Mind in Society. The Development of Higher Psychological Processes. Cambridge, MA: Harvard University Press. 